\title{
Adipokines Do Not Mediate the Association of Obesity and Colorectal Adenoma
}

\author{
Heather M. Ochs-Balcom, ${ }^{1}$ Rikki Cannioto, ${ }^{1}$ Jing Nie, ${ }^{1}$ Amy E. Millen, ${ }^{1}$ Jo L. Freudenheim, ${ }^{1}$ \\ Zhengyi Chen, ${ }^{2}$ Cheryl L. Thompson, ${ }^{2}$ Russell Tracy, ${ }^{3}$ and $\mathrm{Li} \mathrm{Li}^{2}$ \\ ${ }^{1}$ Department of Epidemiology and Environmental Health, School of Public Health and Health Professions, University at Buffalo, \\ Buffalo, NY 14214, USA \\ ${ }^{2}$ Department of Family Medicine and Community Health, Case Western Reserve University, Cleveland, OH 44106, USA \\ ${ }^{3}$ Department of Biochemistry, University of Vermont, Burlington, VT 05405, USA
}

Correspondence should be addressed to Heather M. Ochs-Balcom; hmochs2@buffalo.edu and Li Li; 11134q@rocketmail.com

Received 23 April 2014; Accepted 31 July 2014; Published 13 August 2014

Academic Editor: Jia Chen

Copyright (C) 2014 Heather M. Ochs-Balcom et al. This is an open access article distributed under the Creative Commons Attribution License, which permits unrestricted use, distribution, and reproduction in any medium, provided the original work is properly cited.

\begin{abstract}
Purpose. The association between obesity and colon neoplasia is well established but the underlying biological mechanisms are not fully understood. Rates of both obesity and colon cancer differ by race. Adipokines have been postulated as contributors to the observed association; however, few studies have examined the mediating effect of adipokines on the obesity-colon adenoma association with consideration of racial differences. Methods. We determined prediagnostic levels of adiponectin and leptin in Caucasians (217 cases and 650 controls) and African Americans (175 cases and 378 controls) participating in the Case Transdisciplinary Research on Energetics and Cancer Colon Adenoma Study. We evaluated mediating effects of adiponectin and leptin on the association of abdominal adiposity and colon adenoma separately according to race using mediational pathway analysis. Results. We observed differences in circulating adipokine concentrations by race; African Americans had higher levels of leptin and lower levels of adiponectin than Caucasians for both adenoma cases and controls $(P$ values $<0.001)$. Leptin and adiponectin did not mediate the waist-to-hip ratio (WHR) adenoma association in either group (all Sobel $P$ values $>0.27$ ). Conclusions. We found no evidence that leptin or adiponectin mediates the abdominal obesity-colorectal adenoma pathway. Larger studies on how these associations vary by race, sex, and obesity are needed.
\end{abstract}

\section{Introduction}

Colorectal cancer is the third most common cancer in men and women and the second leading cause of cancer deaths in the United States (US) [1]. Racial disparities in incidence and mortality are well recognized; African Americans disproportionately experience the highest colorectal incidence and mortality rates compared to all other race/ethnic groups in the US [1-3]. It is well recognized that reasons for such disparities are complex and multifactorial. Socioeconomic factors, differential stage at diagnosis, and access to medical care are important factors, but they do not fully account for observed disparities $[4,5]$. Other factors such as nutrition, physical activity, obesity, and cultural factors that influence medical decision making before and after diagnosis are currently under study [6].

Obesity, abdominal obesity in particular, is particularly appealing for study as a contributor to cancer health disparities as a potentially modifiable risk factor. While obesity is positively associated with both colon adenomas and colon cancer [7-10], there is a paucity of published data regarding differential associations of obesity and adenoma polyps with race. We previously showed that waist-to-hip ratio (WHR) in particular is positively associated with colon adenomas for both European and African Americans [11]. Biologic mechanisms for the associations of abdominal adiposity and colon adenoma are not completely understood; mechanisms including inflammation and insulin resistance are currently 
under study $[12,13]$. An additional possible mechanism is mediation by leptin and adiponectin. Further, because of known differences in these adipokines by race [14], examination of these relationships within race groups is important.

Herein we extend our previous work on the association of obesity and adenoma [11, 15] by analyzing whether adiponectin and leptin mediate the relationship between adiposity and colon adenoma in a sample of Caucasians and African Americans.

\section{Methods}

2.1. Study Population. Participants were recruited to the Case Transdisciplinary Research on Energetics and Cancer (TREC) Colon Polyps Study, an ongoing screening colonoscopybased study as previously described [15]. In brief, potential participants scheduled for routine colonoscopy at University Hospitals Case Medical Center and/or affiliated endoscopy centers were initially screened by telephone to determine eligibility. We excluded individuals younger than 30 years of age and those reporting a personal history of any cancer, ulcerative colitis, Crohn's disease, or previous colorectal adenoma, as well as individuals who reported a family history of hereditary nonpolyposis colon cancer or familial adenomatous polyposis. This study was approved by the University Hospitals Case Medical Center and University at Buffalo Institutional Review Boards and all participants provided written informed consent.

Data on demographic characteristics and colon cancer risk factors were collected before the colonoscopy via a phone survey and were based on the lifestyle risk factor questionnaire developed by the colon cancer family registry group (http://www.coloncfr.org/index.php/questionnaires). Participants also completed three additional questionnaires which were developed and validated by the Arizona Diet, Behavior, and Quality of Life Assessment Center [16]: the Arizona Food Frequency, Physical Activity, and Meat Preparation questionnaires. Data on smoking history and NSAID use (ever taken aspirin/ibuprofen at least twice a week for $>1$ month) were also collected as part of the phone survey. On the day of the colonoscopy, a research nurse obtained a fasting blood sample for measurement of leptin and adiponectin and performed anthropometric measurements. Anthropometric data allowed for calculation of body mass index (BMI, $\mathrm{kg} / \mathrm{m}^{2}$ ) and waist-to-hip ratio (WHR).

Our study sample included 1527 Caucasians and African Americans in total, including 421 incident colorectal adenoma cases and 1106 adenoma-free controls. We excluded 95 (6.2\%) participants who were missing leptin and adiponectin measurements and $12(1.0 \%)$ who were missing anthropometric measurements, resulting in a final sample size of 217 Caucasian polyp cases, 650 Caucasian controls, 175 African American polyp cases, and 378 African American controls.

2.2. Laboratory Methods. For measurement of leptin and adiponectin, EDTA plasma samples were assayed with solid phase quantitative sandwich ELISA (R\&D systems, Minneapolis, MN) according to standard protocol. Frozen pools and lyophilized control materials were used in each assay. All assays were carried out blinded to case-control status. Leptin and adiponectin interassay coefficients of variation ranged from 4.9 to $8.1 \%$ and from 10.0 to $11.3 \%$, respectively.

2.3. Statistical Analysis. Descriptive statistics, stratified by case-control status and race, were estimated using $t$-tests for continuous variables and chi-square tests for categorical variables; the $P$ value for statistical significance was determined a priori at less than 0.05 . We developed general linear models to evaluate linear trends in waist-to-hip ratio (WHR) across quartiles of leptin and adiponectin, using quartile cutoffs estimated from the control group with adjustment for age and sex.

We evaluated whether adiponectin and leptin attenuate the association of WHR and adenoma in logistic models stratified by race. We also applied formal Sobel mediation tests using three steps [17]: (1) evaluate the degree to which each adipokine is independently associated with WHR by linear regression with leptin or adiponectin as the dependent variable and WHR as the independent variable, (2) evaluate the degree to which the adipokine is associated with adenoma by logistic regression with adenoma as the dependent variable and leptin or adiponectin as the independent variable (including WHR), and (3) use the estimated regression coefficients and their standard errors from Steps 1 and 2 to calculate the critical ratio and test whether the indirect effect of WHR on adenoma via leptin or adiponectin is significantly different from zero. We used IBM SPSS Version 21 for all analyses [18].

\section{Results}

We observed several differences by race among incident adenoma cases. African American adenoma cases had a higher BMI ( 31.7 versus $29.1 \mathrm{~kg} / \mathrm{m}^{2}$ ), higher frequency of individuals with BMI $>30 \mathrm{~kg} / \mathrm{m}^{2}$ (51\% versus $36 \%$ ), and a higher mean WHR and waist circumference compared to Caucasian adenoma cases (Table 1). With regard to adiponectin and leptin, we observed lower mean values of adiponectin and higher values of leptin and fasting glucose in African American cases compared to Caucasian cases. African American cases were less likely to report a family history of colorectal cancer (17\% versus $27 \%$ for Caucasians) and more likely to be current smokers (35\% versus $13 \%$ for Caucasians). Among adenomafree controls, there were similar differences in measures of obesity, adiponectin and leptin, fasting glucose, insulin, and smoking status between African Americans and Caucasians.

While African American cases were slightly older than African American controls $(P=0.001)$, African American cases and controls were not different in BMI but cases averaged higher WHR compared to controls (0.97 versus 0.93 , $P=0.001)$. Average leptin values were lower $(P=0.006)$ and adiponectin levels were not statistically significantly different $(P=0.36)$ in African American cases compared to African American controls.

Differences between Caucasian cases and controls included age (cases were slightly older than controls, 


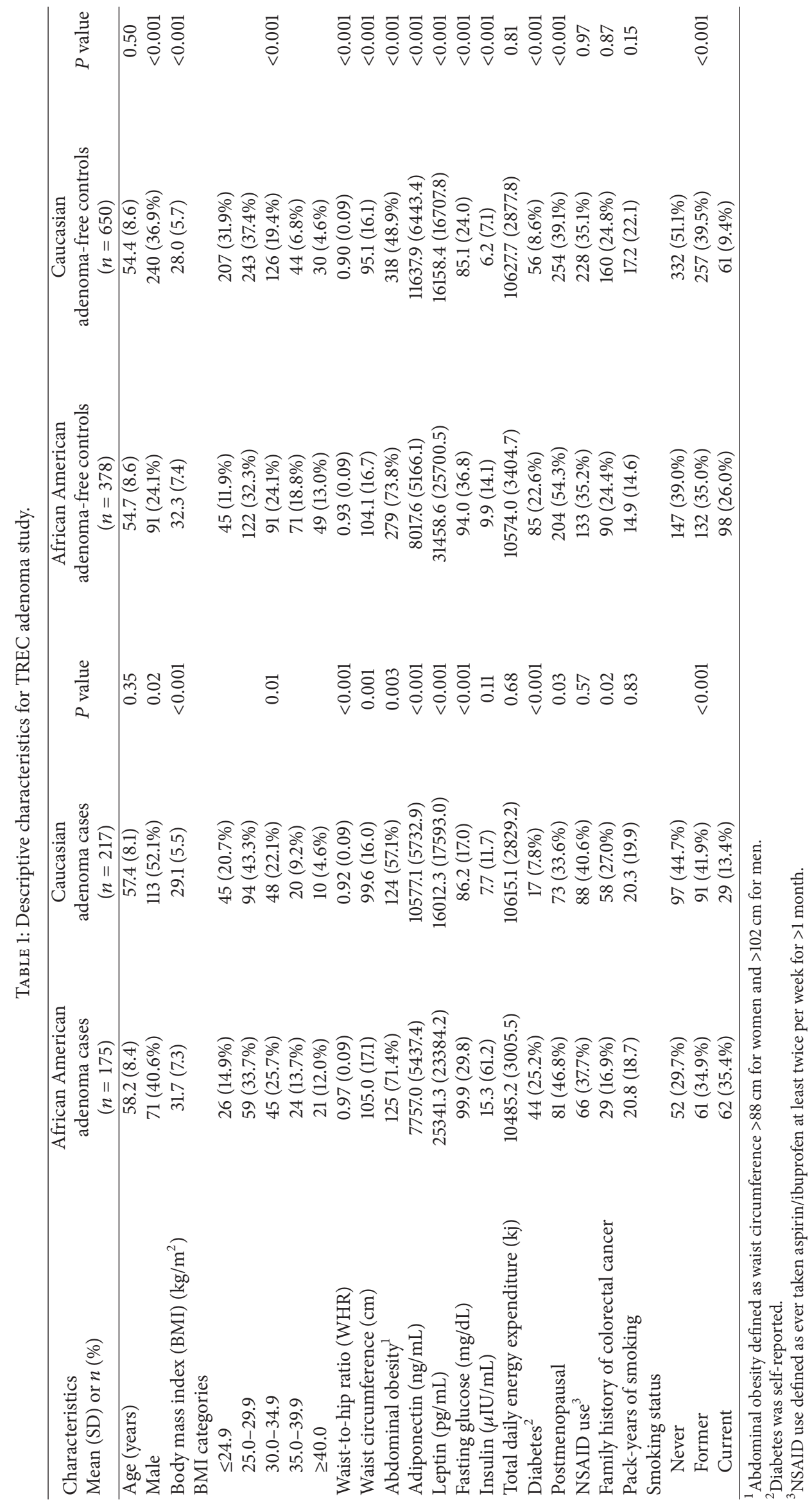


TABLE 2: Race-stratified mediation analysis [17].

\begin{tabular}{|c|c|c|c|c|c|c|}
\hline Race & Biomarker & $\begin{array}{l}\text { Step 1 } \\
\beta(\mathrm{SE})^{1}\end{array}$ & $\begin{array}{l}\text { Step } 1 \\
P \text { value }\end{array}$ & $\begin{array}{l}\text { Step } 2 \\
\beta^{\prime}(\mathrm{SE})^{2}\end{array}$ & $\begin{array}{l}\text { Step } 2 \\
P \text { value }\end{array}$ & $\begin{array}{c}\text { Step } 3 \\
\text { Sobel mediation } \\
P \text { value }\end{array}$ \\
\hline \multirow{2}{*}{ African Americans } & Leptin & $8313.5(11456.5)$ & 0.47 & $-0.000005(0.000005)$ & 0.31 & 0.56 \\
\hline & Adiponectin & $-13743.7(2515.4)$ & $<0.001$ & $0.000004(0.000019)$ & 0.84 & 0.83 \\
\hline \multirow{2}{*}{ Caucasians } & Leptin & $39913.3(7003.6)$ & $<0.001$ & $0.000005(0.000005)$ & 0.36 & 0.32 \\
\hline & Adiponectin & $-14566.5(2497.2)$ & $<0.001$ & $-0.000016(0.000015)$ & 0.30 & 0.29 \\
\hline
\end{tabular}

${ }^{1}$ Linear regression of WHR (independent variable) and adipokine (dependent variable) adjusted for age and sex.

${ }^{2}$ Logistic regression of adipokine (independent variable) and adenoma (dependent variable) adjusted for age, sex, and WHR.

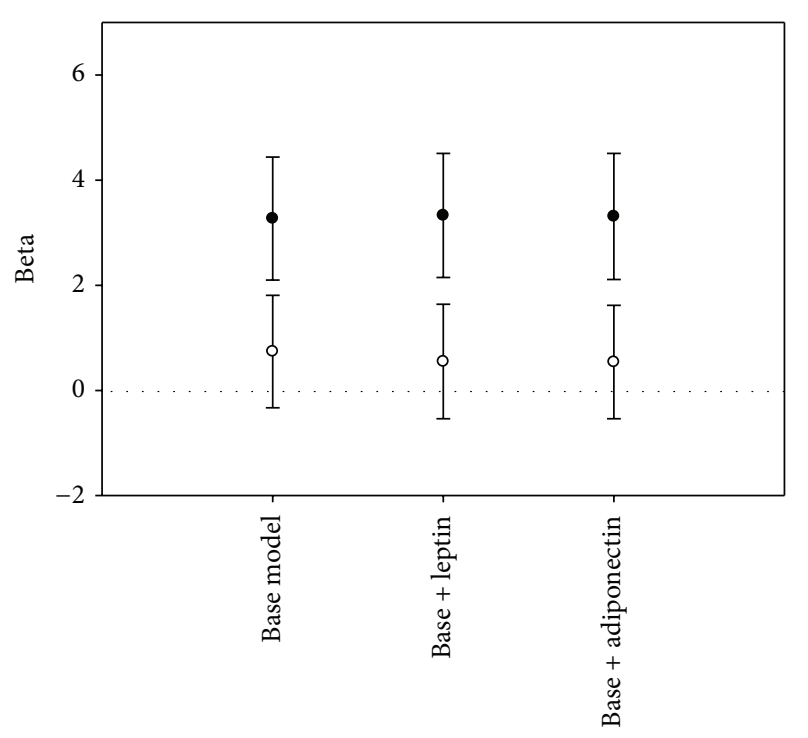

- African Americans

- Caucasians

FIGURE 1: Beta coefficients (95\% confidence intervals) for the association between WHR and adenoma stratified on race and adjusted for age and sex, examining possible mediation by leptin and adiponectin. WHR, leptin, and adiponectin entered as continuous variables.

$P=0.001$ ), BMI (29.1 versus $28.0 \mathrm{~kg} / \mathrm{m}^{2}$, for cases and controls, resp., $P=0.003$ ), and WHR and waist circumference (both $P=0.001)$. We observed no statistically significant differences in mean leptin $(P=0.92)$ or adiponectin $(P=0.07)$ between Caucasian cases and controls.

The association of WHR and adenoma for African Americans and Caucasians, adjusted for age and sex, is shown in Figure 1. These analyses include the hypothesized mediation of leptin and adiponectin. Across both groups it can be seen that adding leptin or adiponectin to the models has negligible or very minimal attenuation on the association of WHR and polyps.

Step 1 of the formal mediation analysis was supportive of an association between WHR and adiponectin for both African Americans and Caucasians, whereas WHR was associated with leptin in Caucasians only (Table 2). In the second step of the mediation analysis, we tested the association of each adipokine with adenoma and we found no statistically significant associations. Using the estimates from Steps 1 and 2 to formally test for mediation, we found no evidence for statistical significance for a mediating effect of adipokine in either African Americans or Caucasians.

\section{Discussion}

We observed several interesting and statistically significant racial differences in adiponectin, as well as associations between abdominal obesity and both adipokines in the direction we expected. The Sobel mediation analysis relies on estimates of the association between obesity (WHR) and adipokines and adenoma status. In our study we observed no associations of either leptin or adiponectin with adenoma status for either Caucasians or African Americans; therefore our study does not support a mediating effect of either leptin or adiponectin in the obesity-adenoma pathway.

The association between circulating leptin and colorectal adenomas has been investigated in three epidemiologic studies [19-21]. Chia et al., 2007, found increased adenoma risk for men but not women with the highest leptin $(\mathrm{OR}=$ 3.3, 95\% CI: $1.2-8.7 ; P$ trend $=0.01$ ); the association was attenuated to $\mathrm{OR}=2.3,95 \% \mathrm{CI}$ : $0.7-7.7$, and was no longer significant with BMI adjustment [19]. This study included primarily Caucasians. The second study, a case-control study of Japanese men and women, reported no association between leptin and adenoma risk when cases and controls were age-, sex-, and BMI-matched [20]. More recently, a case-control study of Japanese men and women reported a statistically significant association between adenoma risk and circulating leptin levels among men, but not women [21]. After adding BMI to the model, ORs were attenuated among men but remained borderline statistically significant ( $\mathrm{OR}=1.44,95 \%$ : 0.99-2.08). These studies are consistent in that adjustment for BMI mitigates an association of leptin and adenoma.

A recent meta-analysis was conducted to analyze the association of circulating leptin and both colorectal adenoma and colorectal cancer [22]. While the individual studies did not show higher relative risk for increasing leptin concentration, the meta-analysis suggested a 35\% increased adenoma risk for the highest versus the lowest leptin categories (RR: 1.35, 95\% CI: 1.03-1.76) and no association of leptin and colorectal cancer. Additional and larger studies of leptin 
and adenoma are needed to obtain more representative and stable estimates and to examine differences within subgroups including sex and race.

Three of the four studies assessing circulating adiponectin and adenoma risk yielded statistically significant inverse associations. In each of three case-control studies of Japanese men and women there was a statistically significant association between circulating adiponectin and adenoma risk, with decreases in odds of adenoma ranging between $44 \%$ and $76 \%$ with every $1 \mu \mathrm{g} / \mathrm{mL}$ increase in total adiponectin $[20,21,23]$. The one prospective study, which included male participants only, did not report statistically significant associations [24]. Furthermore, two studies assessed the relationship between high molecular weight adiponectin and adenomas, but neither study observed statistically significant associations [21, 24].

Two meta-analyses of adiponectin concentrations and colorectal cancer and adenomas were recently published and analyzed similar published literature $[25,26]$. The first meta-analysis included 13 studies totaling 6,175 participants (3015 colorectal cancer cases and/or adenoma cases) [26]. The weighted mean differences between cases and controls were $-1.084 \mu \mathrm{g} / \mathrm{mL}$ (95\% CI: $-1.84-0.33, P=0.05)$ for colorectal cancer cases and $-1.43 \mu \mathrm{g} / \mathrm{mL}$ (95\% CI: $-2.23-$ $0.63, P=0.0001)$ for adenoma cases [26]. Sex-stratified analyses yielded a $2 \%$ decreased risk of colorectal neoplasm for a $1 \mu \mathrm{g} / \mathrm{mL}$ increase in adiponectin (OR $=0.98,95 \%$ CI: 0.96-0.99) among men, but a statistically significant trend was not observed for women (OR $=0.99$, 95\% CI: 0.97 1.01) [26]. These authors concluded that colorectal cancer and adenoma cases have "markedly lower" adiponectin concentrations and, more specifically, that a negative dose-response relationship was evident in men. A second meta-analysis included 2632 cases of colorectal cancer or adenoma and 2753 healthy controls [25]; findings of this meta-analysis suggest that adiponectin levels are statistically significantly lower in patients with colorectal cancer or adenoma compared with controls. A weighted mean difference of -1.51 (95\% CI: -2.42$0.59), P_{\text {heterogeneity }}<0.0001$, was observed in colorectal cancer in comparison to controls, and a weighted mean difference of -1.29 (95\% CI: $-2.01-0.58), P_{\text {heterogeneity }}<0.0001$, was observed in adenoma cases [25]. The authors cautioned that case-control studies with smaller sample sizes yielded the greatest mean differences and that additional larger-scale epidemiological studies were warranted [25].

To date, one study assessed the role of adipokines as potential mediating factors in the obesity-colorectal cancer pathway [27]. In this study of postmenopausal women, Ho et al. found that leptin, and not adiponectin, mediated the association between abdominal obesity and colorectal cancer risk. Their primary outcome of interest was colorectal cancer, whereas our outcome was adenoma. Their sample was comprised of primarily white (postmenopausal) women while our study represents adult Caucasian and African American men and women. The current literature and our own data suggest that postmenopausal women have higher leptin concentrations. The obesity-colorectal cancer association may be more strongly influenced by adipocyte dysfunction and adipokine dysregulation, and the mechanism for the obesity-precancerous adenoma pathway, initiated as much as 10 years earlier, may be different.

Much of the epidemiological data examining the obesityadipokine-colorectal cancer pathway has been conducted with Asian populations, and very few studies have been published using US samples where obesity rates may be higher. To our knowledge, ours is the first US-based epidemiological study including a large sample of African Americans; it is possible that differences in findings may be reflective of both environmental and racial differences related to obesity which have not yet been fully elucidated.

Epidemiological evidence suggests that circulating plasma concentrations of adiponectin and leptin may not precisely reflect an individual's true or long-term levels $[14,27]$. Also, for certain adipokines, the adipokine tissue concentrations in the tumor environment may be more relevant to the assessment of cancer related risk than circulating blood levels. Recent epidemiological evidence has suggested only a moderate correlation between plasma and breast tissue leptin and a weak correlation between plasma adiponectin and breast tissue adiponectin [14]; it is not known if there is a similar lack of correlation between blood and colon tissue concentrations. This discordance between plasma and tissue concentrations of adiponectin and leptin may, in part, explain why some studies have not observed associations between blood concentrations of adipokines and colorectal cancer risk. Finally, relying on total adiponectin, as opposed to identifying more bioactive components of adiponectin, may also explain some of the inconsistencies in the literature and our null findings.

Our study provides new important data because it includes a large sample of both African American and Caucasian participants. To our knowledge, this is the largest and most diverse US epidemiologic study of the relationship between obesity, adiponectin, leptin, and adenoma. Additional strengths of the study include the direct measurement of anthropometric variables by study nurses and the inclusion of data representing all well-established risk and protective factors associated with colorectal cancer (i.e., smoking, NSAID use, alcohol, physical activity, family history, and several measures of adiposity).

Weaknesses of the study include the reliance on a single measure of each of the adipokines taken from blood samples collected at the time of colonoscopy. Furthermore, reliance upon total adiponectin (as opposed to high molecular versus low molecular weight adiponectin concentrations) could be a limitation given recent evidence suggesting that the high molecular weight and low molecular weight components of adiponectin may have different biological functions. Lastly, reduced sample sizes for subset analyses may have limited our power to detect statistically significant associations.

\section{Conclusions}

Our study is in agreement with previous evidence that circulating blood concentrations of adiponectin and leptin vary by race and obesity. We did not find evidence of a mediating effect of leptin or adiponectin in the 
obesity-adenoma association. A better understanding of the association between obesity, adiponectin, and leptin and adenoma and how these associations vary by race, sex, and obesity is needed to further understand the role that adipokines, recognized as important biologic components of the obesity phenotype, may play in association with cancer risk.

\section{Conflict of Interests}

The authors declare that there is no conflict of interests regarding the publication of this paper.

\section{Authors' Contribution}

Heather M. Ochs-Balcom and Rikki Cannioto contributed equally to this work.

\section{Acknowledgment}

Research in this paper was supported by the National Cancer Institute of the National Institutes of Health under Awards nos. K07CA136969 to Heather M. Ochs-Balcom and U54CA116867 to Li Li.

\section{References}

[1] R. Siegel, D. Naishadham, and A. Jemal, "Cancer statistics, 2012," CA Cancer Journal for Clinicians, vol. 62, no. 1, pp. 10-29, 2012.

[2] L. X. Clegg, M. E. Reichman, B. A. Miller et al., "Impact of socioeconomic status on cancer incidence and stage at diagnosis: selected findings from the surveillance, epidemiology, and end results: national longitudinal mortality study," Cancer Causes and Control, vol. 20, no. 4, pp. 417-435, 2009.

[3] E. Ward, A. Jemal, V. Cokkinides et al., "Cancer disparities by race/ethnicity and socioeconomic status," $C A-A$ Cancer Journal for Clinicians, vol. 54, no. 2, pp. 78-93, 2004.

[4] R. M. Mayberry, R. J. Coates, H. A. Hill et al., "Determinants of black/white differences in colon cancer survival," Journal of the National Cancer Institute, vol. 87, no. 22, pp. 1686-1693, 1995.

[5] B. N. Polite, J. J. Dignam, and O. I. Olopade, "Colorectal cancer and race: Understanding the differences in outcomes between African Americans and whites," Medical Clinics of North America, vol. 89, no. 4, pp. 771-793, 2005.

[6] S. Dein, "Explanatory models of and attitudes towards cancer in different cultures," The Lancet Oncology, vol. 5, no. 2, pp. 119124,2004

[7] S. C. Larsson and A. Wolk, "Obesity and colon and rectal cancer risk: a meta-analysis of prospective studies," The American Journal of Clinical Nutrition, vol. 86, no. 3, pp. 556-565, 2007.

[8] A. A. Moghaddam, M. Woodward, and R. Huxley, "Obesity and risk of colorectal cancer: a meta-analysis of 31 studies with 70,000 events," Cancer Epidemiology Biomarkers and Prevention, vol. 16, no. 12, pp. 2533-2547, 2007.

[9] Q. Ben, W. An, Y. Jiang et al., "Body mass index increases risk for colorectal adenomas based on meta-analysis," Gastroenterology, vol. 142, no. 4, pp. 762-772, 2012.

[10] K. Okabayashi, H. Ashrafian, H. Hasegawa et al., "Body mass index category as a risk factor for colorectal adenomas: a systematic review and meta-analysis," The American Journal of Gastroenterology, vol. 107, no. 8, pp. 1175-1185, 2012.

[11] C. L. Thompson, N. A. Berger, A. Chak, and L. Li, "Racial differences in measures of obesity and risk of colon adenoma," Obesity, vol. 20, no. 3, pp. 673-677, 2012.

[12] R. C. van Kruijsdijk, E. van der Wall, and F. L. Visseren, "Obesity and cancer: the role of dysfunctional adipose tissue," Cancer Epidemiology Biomarkers and Prevention, vol. 18, no. 10, pp. 2569-2578, 2009.

[13] E. E. Calle and R. Kaaks, "Overweight, obesity and cancer: epidemiological evidence and proposed mechanisms," Nature Reviews Cancer, vol. 4, no. 8, pp. 579-591, 2004.

[14] A. A. Llanos, R. G. Dumitrescu, C. Marian et al., "Adipokines in plasma and breast tissues: Associations with breast cancer risk factors," Cancer Epidemiology Biomarkers and Prevention, vol. 21, no. 10, pp. 1745-1755, 2012.

[15] A. P. Ortiz, C. L. Thompson, A. Chak, N. A. Berger, and L. $\mathrm{Li}$, "Insulin resistance, central obesity, and risk of colorectal adenomas," Cancer, vol. 118, no. 7, pp. 1774-1781, 2012.

[16] M. E. Martínez, J. R. Marshall, E. Graver et al., "Reliability and validity of a self-administered food frequency questionnaire in a chemoprevention trial of adenoma recurrence," Cancer Epidemiology Biomarkers and Prevention, vol. 8, no. 10, pp. 941946, 1999.

[17] M. E. Sobel, "Asymptotic confidence intervals for indirect effects in structural equation models," in Sociological Methodology, S. Leinhardt, Ed., pp. 290-312, American Sociological Association, Washington, DC, USA, 1982.

[18] IBM Corp, IBM SPSS Statistics for Windows, IBM Corp, Armonk, NY, USA, 2011.

[19] V. M. Chia, P. A. Newcomb, J. W. Lampe et al., "Leptin concentrations, leptin receptor polymorphisms, and colorectal adenoma risk," Cancer Epidemiology Biomarkers and Prevention, vol. 16, no. 12, pp. 2697-2703, 2007.

[20] T. E. Nakajima, Y. Yamada, T. Hamano et al., "Adipocytokines as new promising markers of colorectal tumors: Adiponectin for colorectal adenoma, and resistin and visfatin for colorectal cancer," Cancer Science, vol. 101, no. 5, pp. 1286-1291, 2010.

[21] T. Yamaji, M. Iwasaki, S. Sasazuki, and S. Tsugane, "Interaction between adiponectin and leptin influences the risk of colorectal adenoma," Cancer Research, vol. 70, no. 13, pp. 5430-5437, 2010.

[22] S. P. Gialamas, T. N. Sergentanis, C. N. Antonopoulos, N. Dessypris, G. P. Chrousos, and E. T. Petridou, "Circulating leptin levels and risk of colorectal cancer and adenoma: a casecontrol study and meta-analysis," Cancer Causes and Control, vol. 24, no. 12, pp. 2129-2141, 2013.

[23] S. Otake, H. Takeda, Y. Suzuki et al., "Association of visceral fat accumulation and plasma adiponectin with colorectal adenoma: evidence for participation of insulin resistance," Clinical Cancer Research, vol. 11, no. 10, pp. 3642-3646, 2005.

[24] J. Fukumoto, T. Otake, O. Tajima et al., "Adiponectin and colorectal adenomas: Self Defense Forces Health Study," Cancer Science, vol. 99, no. 4, pp. 781-786, 2008.

[25] W. An, Y. Bai, S.-X. Deng et al., "Adiponectin levels in patients with colorectal cancer and adenoma: a meta-analysis," European Journal of Cancer Prevention, vol. 21, no. 2, pp. 126-133, 2012.

[26] X. T. Xu, Q. Xu, J. L. Tong et al., "Meta-analysis: circulating adiponectin levels and risk of colorectal cancer and adenoma," Journal of Digestive Diseases, vol. 12, no. 4, pp. 234-244, 2011.

[27] G. Y. F. Ho, T. Wang, M. J. Gunter et al., "Adipokines linking obesity with colorectal cancer risk in postmenopausal women," Cancer Research, vol. 72, no. 12, pp. 3029-3037, 2012. 


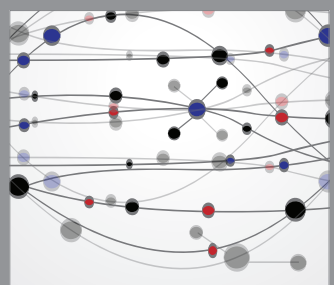

The Scientific World Journal
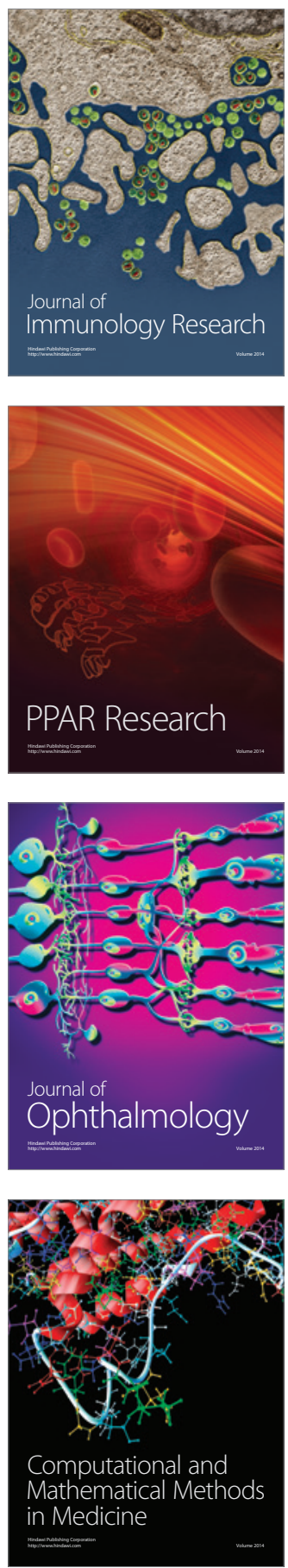

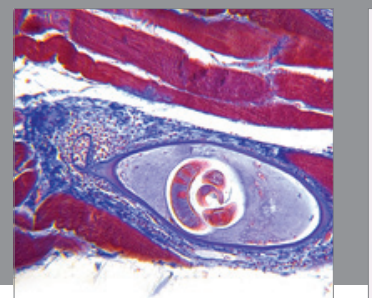

Gastroenterology

Research and Practice
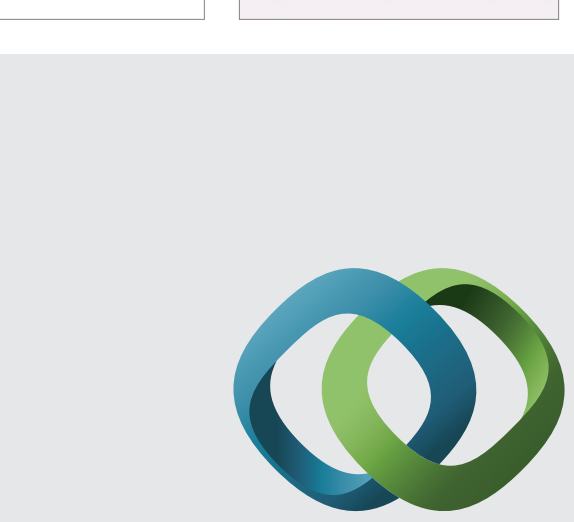

\section{Hindawi}

Submit your manuscripts at

http://www.hindawi.com
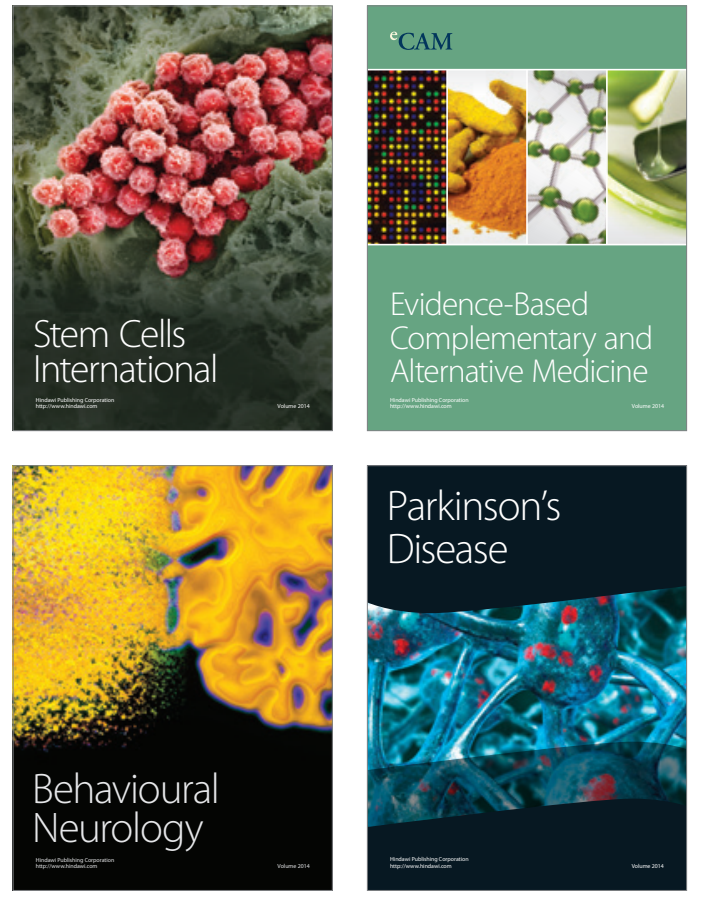
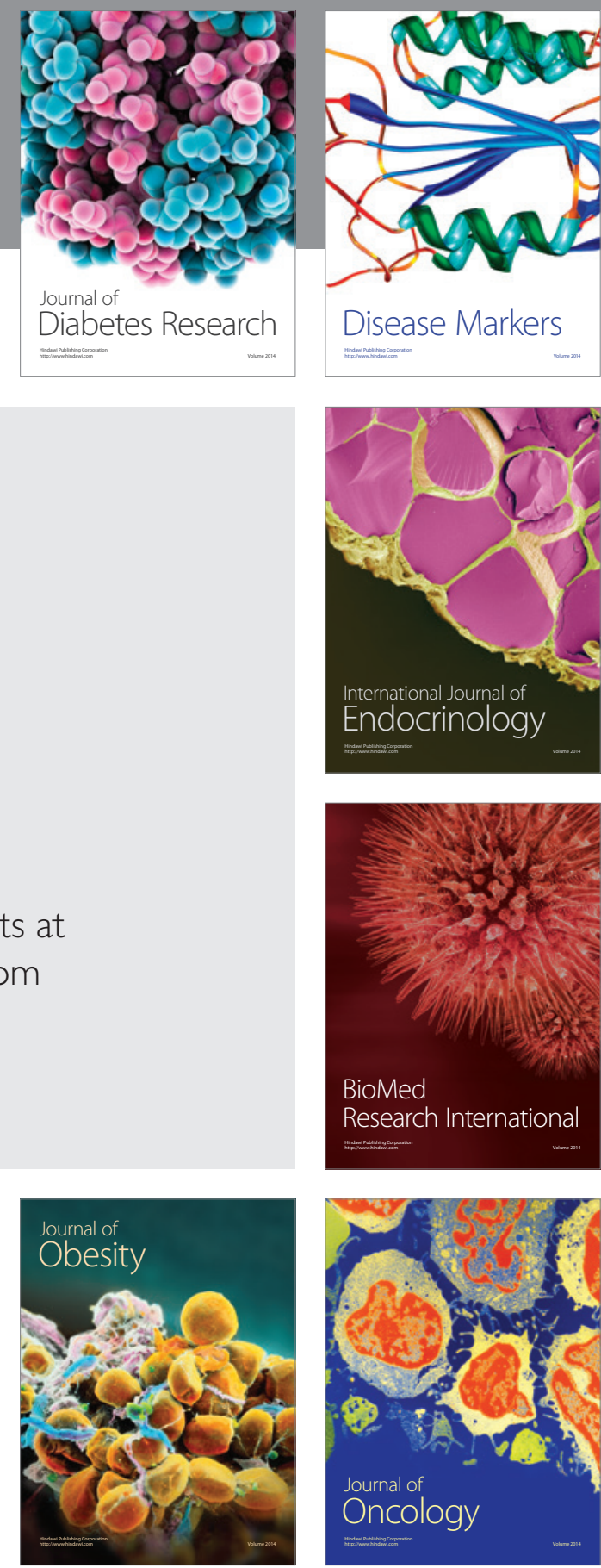

Disease Markers
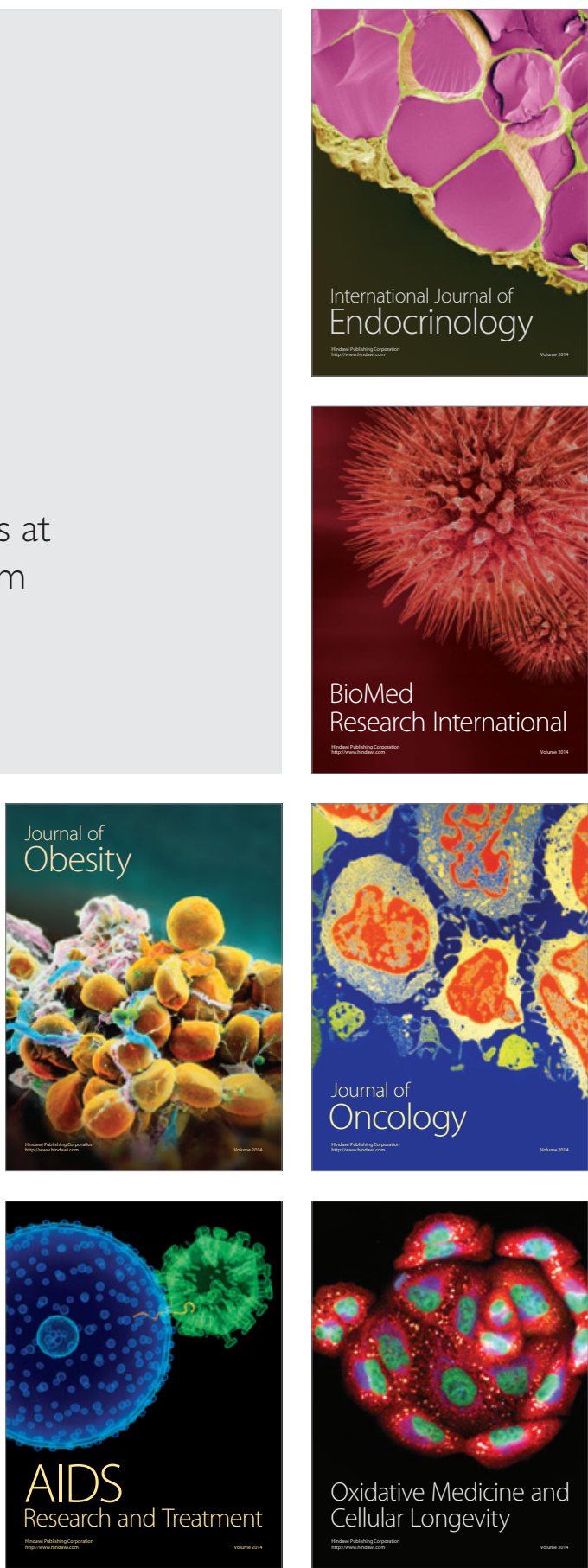